\title{
Characterization of tumor microvascular structure and permeability: comparison between magnetic resonance imaging and intravital confocal imaging
}

\author{
Nina Kristine Reitan \\ Norwegian University of Science and Technology \\ Department of Physics \\ Høgskoleringen 5 \\ N-7491 Trondheim, Norway
}

\begin{abstract}
Marte Thuen
Norwegian University of Science and Technology Department of Circulation and Medical Imaging N-7489 Trondheim, Norway
\end{abstract}

Pål Erik Goa

St. Olav's Hospital

Department of Medical Imaging

N-7006 Trondheim, Norway

\section{Catharina de Lange Davies}

Norwegian University of Science and Technology Department of Physics

Høgskoleringen 5

N-7491 Trondheim, Norway

\begin{abstract}
Solid tumors are characterized by abnormal blood vessel organization, structure, and function. These abnormalities give rise to enhanced vascular permeability and may predict therapeutic responses. The permeability and architecture of the microvasculature in human osteosarcoma tumors growing in dorsal window chambers in athymic mice were measured by confocal laser scanning microscopy (CLSM) and dynamic contrast enhanced magnetic resonance imaging (DCE-MRI). Dextran (40 kDa) and Gadomer were used as molecular tracers for CLSM and DCE-MRI, respectively. A significant correlation was found between permeability indicators. The extravasation rate $K_{i}$ as measured by CLSM correlated positively with DCE-MRI parameters, such as the volume transfer constant $K^{\text {trans }}$ and the initial slope of the contrast agent concentration-time curve. This demonstrates that these two techniques give complementary information. Extravasation was further related to microvascular structure and was found to correlate with the fractal dimension and vascular density. The structural parameter values that were obtained from CLSM images were higher for abnormal tumor vasculature than for normal vessels. ๑ 2010 Society of Photo-Optical Instrumentation Engineers. [DOI: 10.1117/1.3431095]
\end{abstract}

Keywords: confocal laser scanning imaging; magnetic resonance imaging; dorsal window chamber; permeability; vasculature.

Paper 09571R received Dec. 23, 2009; revised manuscript received Mar. 10, 2010; accepted for publication Mar. 15, 2010; published online Jun. 16, 2010.

\section{Introduction}

Tumor blood supply plays a critical role in cancer therapy. Flow rate, vascular structure, and permeability are responsible for the hypoxic and acidic tumor microenvironment. ${ }^{1,2}$ The vasculature and microenvironment determine the efficacy of radiotherapy, chemotherapy, and drug delivery to solid tumors. ${ }^{3-9}$ Most tumors develop their vascular network by angiogenesis, and tumor vasculature differs markedly from that of the host tissue. Tumor vasculature has an abnormal structure and function and a high geometric resistance to blood flow, leading to a spatially and temporally heterogeneous blood flow rate. ${ }^{2,10-12}$

The heterogeneity of the tumor vasculature also includes the vessel walls. In general, the capillary wall is poorly developed, with fenestrae between endothelial cells, a discontinuous endothelial cell layer, and a partially absent basal lamina, resulting in leaky blood vessels. However, parts of the vessel walls may also resemble normal vessels, contributing to heterogeneous vessel permeability. High permeability favors the transcapillary transport of therapeutic macromolecules, ${ }^{2,13}$ which is beneficial for delivery of nanomedicines such as proteins and encapsulated drugs or genes. However, although ex-

Address all correspondence to: Nina Kristine Reitan, Norwegian University of Science and Technology, Department of Physics, Høgskoleringen 5, Trondheim, 7491 Norway Tel: 47-73-598-709; Fax: 47-73-597-710; E-mail: nina.reitan@ntnu.no travasation of macromolecules in tumors may be enhanced relative to normal vessels, the elevated interstitial fluid pressure in tumors ${ }^{14,15}$ reduces the transvascular pressure gradient and thereby the fluid flow from the vessels to the interstitial space. Due to the high interstitial fluid pressure in tumors, diffusion becomes the major transport mechanism.

Another known characteristic of tumors is the highly chaotic structure of the blood vessels, which prevents adequate perfusion of the tissue ${ }^{16}$ and inhibits treatment. The tumor vasculature consists of complex branching patterns, tortuous and bending vessels, and a heterogeneous spatial distribution. Vascular density and fractal dimension can be used to determine the tumor vascular structure. ${ }^{17,18}$ Fractal dimension describes irregular, tortuous contours and branching structures, which are characteristic of tumor microvasculature, and it has been shown that the fractal dimension is higher for tumors than for normal microvasculature. ${ }^{19,20}$

The crucial effect of vasculature on the microenvironment and therapeutic outcomes illustrates the need to develop methods to study vascular structure and permeability. Magnetic resonance imaging (MRI) is a noninvasive imaging technique that is commonly used to study vascular structure and function. ${ }^{21-23}$ Tracer kinetic analysis of contrast-enhanced images allows the estimation of physiological parameters such as blood volume, blood flow, and vessel permeability. ${ }^{24}$ How-

$1083-3668 / 2010 / 15(3) / 036004 / 11 / \$ 25.00$ (C) 2010 SPIE 
ever, MRI does not possess sufficient spatial resolution to visualize cellular and subcellular structures. Intravital optical microscopy using confocal laser scanning or two-photon microscopy has superior spatial resolution, and several cellular and extracellular characteristics can be imaged simultaneously and colocalized in three dimensions. ${ }^{25,26}$

The purpose of the present work was to compare vascular parameters obtained with confocal laser scanning microscopy (CLSM) and dynamic contrast-enhanced MRI (DCE-MRI) to investigate how these techniques might complement each other. The heterogeneous tumor microvasculature induces a high degree of uncertainty in the quantification of structural and transport parameters. This uncertainty can be reduced through validation and cross-comparison of results obtained by the two imaging techniques. The dorsal window chamber allows direct examination of in vivo tumor angiogenesis and microcirculation over several days. Tumors were grown in dorsal window chambers in athymic mice, and microvasculature and vascular permeability to $40-\mathrm{kDa}$ dextran and Gadomer were studied by CLSM and DCE-MRI, respectively.

\section{Materials and Methods}

\subsection{Animals and Tumor Model}

Dorsal window chambers in an MRI-compatible material (POM: poloxy methylene) were produced. The total weight of the chamber (including the window) was $2.0 \mathrm{~g}$ and exerted less strain on the animals than its heavier titanium counterpart. The chambers were implanted on the backs of athymic female BALB/c nu/nu mice (7 to 9 weeks old,) weighing 23 to $25 \mathrm{~g}$ each (Taconic, M\&M, Ry, Denmark), as described previously. ${ }^{26}$ At $24 \mathrm{~h}$ after implanting the chambers, the coverslips were removed, and $1.5 \times 10^{6}$ cells from the human osteosarcoma cell line $\mathrm{OHS}^{27}$ were placed into the center of each chamber. New glass coverslips were placed over the areas, and the chambers were closed. The xenografts were allowed to grow for two weeks before starting the experiments. During this period, the animals were given $64 \mathrm{mg} / \mathrm{kg} /$ day Baytril (enrofloxacin) in drinking water containing $2 \%$ sucrose. At the time of the experiments, the tumors were approximately $0.5 \mathrm{~mm}$ thick and filled the entire window area. The mice were anesthetized by subcutaneous injections of fentanyl/midazolam/Haldol/sterile water $(3: 3: 2: 4)$ at a dose of $12 \mathrm{ml} / \mathrm{kg}$ body weight (Hameln Pharmaceuticals, Hameln, Germany; Alpharma AS, Oslo, Norway; and Janssen-Cilag AS, Oslo, Norway). The tail vein was cannulated to allow injection of molecular tracers. Time series images were acquired from the same animal by CLSM (day 1) and DCE-MRI (day 2). Mice with dorsal window chambers but no implanted tumors were used to study normal tissue vasculature. Parameters were calculated from images of six mice with tumor and six controls. In addition, one mouse with a tumor was used for high-resolution MR imaging of blood vessels. The animals were kept under pathogen-free conditions at a constant temperature of 19 to $22{ }^{\circ} \mathrm{C}, 50$ to $60 \%$ humidity, and 65 air changes per h, and animals were allowed food and water $a d$ libitum. The surgical procedures were performed under sterile conditions. All animal experiments were carried out with ethical committee approval.

\subsection{Tracers}

\subsubsection{CLSM}

For permeability measurements, 40-kDa TMR (tetramethylrhodamine)-dextran (Sigma-Aldrich, St. Louis, Missouri) was used as a fluorescent tracer, as this molecule has approximately the same molecular weight as the MR contrast agent Gadomer (see below). TMR is photostable and suitable for dynamic imaging. FITC (fluorescein isothiocyanate)-dextran (2 MDa) (Sigma-Aldrich, St. Louis, Missouri) was used as a blood pool marker.

\subsubsection{MRI}

The contrast agent Gadomer (Bayer Schering Pharma AG, Berlin, Germany) was used for estimating permeability. Gadomer contains $24 \mathrm{Gd}$ ions at the surface of a dendritic backbone and has a molecular weight of approximately $17 \mathrm{kDa}$. Due to its globular shape, however, the apparent molecular weight of Gadomer is 30 to $35 \mathrm{kDa},{ }^{28}$ which is large enough to ensure slow diffusion through the vascular wall. ${ }^{29}$ Gadomer has low toxicity and is primarily eliminated by glomerular filtration. ${ }^{28}$ Albumin-(Gd-DTPA) (92 kDa) was synthesized based on a procedure originally described by $\operatorname{Ogan}^{30}$ and applied to MR angiography of blood vessels.

\subsection{Confocal Laser Scanning Microscopy}

\subsubsection{Experimental setup}

The animal was anesthetized. To locate the tumor, an overview image of the tissue was obtained using a stereomicroscope (Motic SMZ-168 Stereo Zoom Microscope, Motic Deutschland GmbH, Wetzlar, Germany), showing that the tissue in the entire chamber window area in general comprised irregular, neoformed vessels.

The tail vein was cannulated, and $100 \mu \mathrm{l}$ of 2-MDa FITCdextran $(20 \mathrm{mg} / \mathrm{ml})$ was injected to visualize the vasculature. The cannula was flushed with $250 \mathrm{U} / \mathrm{ml}$ heparin (Sigma Chemical Co., St. Louis, Missouri) to avoid blood clotting, and the animal was immediately placed on the microscope stage (LSM Meta 510, Carl Zeiss Jena GmbH, Jena, Germany) for imaging. The animal's body temperature was kept at $37^{\circ} \mathrm{C}$. Images with a field of view of 460.7 $\times 460.7 \mu \mathrm{m}^{2}$ and $512 \times 512$ pixel resolution (pixel size 0.9 $\times 0.9 \mu \mathrm{m}^{2}$ ) were acquired using a Plan-Neofluar $20 \times / 0.5$ objective. For confocal imaging of microvascular structures, Z-stacks of blood vessels were acquired using a 488-nm laser, a BP500 550-nm filter in front of the detector, and a pinhole diameter of $94 \mu \mathrm{m}$. For each tumor, extravasation of $40-\mathrm{kDa}$ TMR-dextran was measured in three separate locations containing microvessels to address the heterogeneity of the tumor vasculature. Background images before injection and time series images after injection of $100 \mu \mathrm{l}$ of $40-\mathrm{kDa}$ TMR-dextran $(30 \mathrm{mg} / \mathrm{ml})$ were acquired for each location using the $543-\mathrm{nm}$ laser, an LP560 filter in front of the detector, and a pinhole diameter of $86 \mu \mathrm{m}$. The time series images of the three locations were acquired successively with a mean time interval of approximately $20 \mathrm{~s}$ for the first $10 \mathrm{~min}$ and less frequently thereafter, for a total time period up to $60 \mathrm{~min}$. The injection time of both tracers was $5 \mathrm{~s}$. 


\subsubsection{Image post-processing and data analysis}

To minimize contributions to the signal from sources other than the molecular tracer, a background image was taken before injection of 40-kDa dextran and subtracted from the time series images acquired post-injection. Intra- and extravascular image analyses were performed by applying motion-corrected vascular masks to the time series images. The total field of view was considered to be the region of interest (ROI). Blood vessel contours were manually traced, and the extravascular area in the ROI was defined as the space at a distance of more than $4 \mu \mathrm{m}$ from the blood vessel walls. By visual inspection, a distance of $4 \mu \mathrm{m}$ was considered sufficient to avoid fluorescence contribution from the intravascular area. Fluorescence intensity as a function of time was measured for both intra- and extravascular areas using the ImageJ (version 1.38, available from the NIH, http://rsb.info.nih.gov/ij/) Time Series Analyzer plug-in.

Vasculature permeability was estimated using the method described by Patlak et al. ${ }^{31}$ This method is based on plots of the ratio of extravascular to intravascular concentration of the tracer against a modified time axis (Patlak plot). The concentration was assumed to be proportional to the fluorescence intensity. The values along the ordinate axis are given by ${ }^{32}$

$$
y=\frac{C_{t}(t)}{C_{\text {vess }}(t)},
$$

where $C_{t}(t)$ and $C_{\text {vess }}(t)$ are the extra- and intravascular concentrations at time $t$. The modified time axis is defined by ${ }^{32}$

$$
T=\frac{\int C_{\text {vess }}(t) \mathrm{d} t}{C_{\text {vess }}(t)},
$$

where the concentration in the numerator is integrated over time from injection of the tracer. When there is a net irreversible accumulation of fluorescence in the tissue over the time course of the experiment, the plot becomes linear after equilibrium is reached. The terminal slope of the straight line is equal to the rate constant describing the net irreversible transport of the fluorescent tracer from the capillaries to the extravascular space. The extravasation rate $K_{i}$ was estimated by fitting an exponential function to the extravascular/ intravascular intensity ratio plotted as a function of $T:^{32}$

$$
y(T)=\left(\alpha+K_{i} T\right)\left(1-e^{-\gamma T}\right)
$$

where $\alpha$ and $\gamma$ are constants. When $T$ is large, $y(T)$ increases linearly with $T$, with a positive slope $K_{i}{ }^{31}$ By visual inspection, the first 35 min of measurements were considered sufficient to fulfill the requirement of a net irreversible accumulation.

Under permeability-limited conditions, $K_{i}$ equals the permeability surface area product. ${ }^{33}$ Following the approach of Reyes-Aldasoro et al., ${ }^{32}$ the extravasation rate can be expressed as

$$
K_{i}=\frac{P S}{V_{t}},
$$

where $P$ is apparent permeability, and $S$ is the vascular surface area contained in the imaged tissue volume $V_{t}$. All ROIs imaged by CLSM were of equal size, and $V_{t}$ is therefore assumed constant.

$S$ and other vascular morphology parameters (the number of branching points and the vascular density) were estimated using the software Amira (Visage Imaging $\mathrm{GmbH}$, Berlin, Germany) after tracing the blood vessels with the filament editor. The number of branching points was defined as the number of vessel nodes in the filament-traced image and is an estimate of the number of neo-formed vessels. The vascular density was calculated as the fraction of the image area that was covered by vessels. ${ }^{17}$ The surface area $S$ was calculated as the sum of the surface areas of all vessels in the ROI. For blood vessels with diameters larger than the detected optical section $(4.5 \mu \mathrm{m})$, the vessel shape was approximated as a rectangular prism with a depth of $4.5 \mu \mathrm{m}$ instead of a cylinder.

The fractal dimension was determined using Fractal Box Count in ImageJ, which counts the number of boxes of an increasing size needed to cover a one-pixel binary object boundary. The fractal dimension is calculated from the slope (fractal dimension $=-$ slope) of a log-log plot of the box size and the number of boxes necessary to cover the boundary. The method was verified by comparing measured fractal dimensions of known fractals to theoretical values, which were found to be in good agreement. Fractal theory and its relevance for vascular networks have been described previously. ${ }^{17,18}$

\section{$2.4 M R I$}

\subsubsection{Experimental setup}

MRI was performed at 7T using a Bruker Biospec Avance 70/20 (Bruker Biospin, Ettlingen, Germany) with a 72-mm-volume coil for transmission and an actively decoupled quadrature rat head surface coil for receive-only. The animals were anesthetized, cannulated, and placed in a prone position in the magnet on a dedicated animal bed with the chamber positioned in the isocenter. The body temperature was kept at 37 to $38^{\circ} \mathrm{C}$ with circulating water within the animal bed. Pilot scans were applied to localize the appropriate tissue within the chamber and to align the slice plane with the chamber.

For blood vessel imaging, $200 \mu \mathrm{l}$ of albumin-(Gd-DTPA) (20 mg/ml) was injected. A $\mathrm{T}_{1}$-weighted 3-D Flash (fast, low flip angle shot) sequence was applied with $\mathrm{TR}=15 \mathrm{~ms}$, TE $=2.4 \mathrm{~ms}$, and flip angle $=25.0 \mathrm{deg}$. The voxel size was 145 $\times 145 \times 144 \mu^{3}$, and the acquisition time was $16.38 \mathrm{~min}$.

For DCE-MRI, a $\mathrm{T}_{1}$ map was obtained using a turbo spinecho sequence (RARE-VTR) with $\mathrm{TR}=150,600,1200,3000$, and $10,000 \mathrm{~ms} ; \mathrm{TE}=12.5 \mathrm{~ms}$; and RARE-factor $=2$. One slice of a thickness $0.3 \mathrm{~mm}$ was imaged at a resolution of $578 \times 578 \mu \mathrm{m}^{2}$, and the acquisition time was $7 \mathrm{~min}, 58 \mathrm{~s}$. The dynamic series was acquired using a $\mathrm{T}_{1}$-weighted spinecho RARE sequence with $\mathrm{TR}=200 \mathrm{~ms}, \mathrm{TE}=7 \mathrm{~ms}$, two averages, and five slices of thickness $0.3 \mathrm{~mm}$. The field of view was $37 \times 37 \mathrm{~mm}^{2}$, with $64 \times 64$ pixel resolution (pixel size 
$578 \times 578 \mu \mathrm{m}^{2}$ ) and 350 repetitions. The time resolution was $6.4 \mathrm{~s}$, with a total acquisition time of $37 \mathrm{~min}, 20 \mathrm{~s}$. Gadomer $(0.2 \mathrm{mmol} / \mathrm{kg})$ was injected during the ninth repetition, over a period of $10 \mathrm{~s}$.

\subsubsection{Data analysis}

DCE-MRI data were analyzed using in-house software written in MATLAB R2007b (MathWorks, Inc., Natick, Massachusetts). Native $T_{1,0}$ before contrast injection was estimated using a least-squares fitting of the multi-TR recording to the standard spin-echo signal equation. The mean value of $T_{1}$ for all tumors was used as input into the further analysis. An ROI was defined from post-contrast images as the volume in the chamber window where signal enhancement was detected, and the signal intensity inside the ROI was averaged. At each time point, the effective $T_{1}$ was calculated using the spin-echo equation, and the absolute concentration was then calculated using the following equation:

$$
C_{t}=\frac{\left(\frac{1}{T_{1}}-\frac{1}{T_{1,0}}\right)}{r},
$$

where $r$ is the relaxivity of Gadomer, which at 7T was set to $7 \mathrm{mM}^{-1} \mathrm{~s}^{-1}$. $^{34}$ A two-compartment kinetic model developed by Tofts et al. ${ }^{24}$ was fitted to the concentration curves. Tofts's model assumes that the tissue consists of a plasma compartment and an extravascular extracellular space (EES) and that the rate constants $k_{p e}$ (transfer rate from blood plasma to EES) and $k_{e p}$ (transfer rate from EES to blood plasma) are identical. The volume transfer constant $K^{\text {trans }}$ is related to $k_{e p}$ by ${ }^{24}$

$$
k_{e p}=K^{\text {trans }} / v_{e},
$$

where $v_{e}$ is the volume of the EES per unit volume of tissue. $K^{\text {trans }}$ and $k_{e p}$ have the same units $\left(\mathrm{min}^{-1}\right)$, but $k_{e p}$ is derived from the shape of the concentration-time curve, whereas $K^{\text {trans }}$ requires absolute values of the tracer concentration. $K^{\text {trans }}$ is a commonly used permeability indicator that is related to the extravasation rate $K_{i}$ by the definitions used in the present paper and was therefore chosen for comparison to the CLSM data.

The general transport equation is given by ${ }^{24}$

$$
\frac{\mathrm{d} C_{t}}{\mathrm{~d} t}=K^{\text {trans }}\left(C_{p}-C_{t} / v_{e}\right),
$$

where $C_{t}$ and $C_{p}$ are the tissue and plasma concentrations, respectively. The concentration $C_{p}(t)$ at time $t$ of the contrast agent in the plasma compartment (also called the arterial input function) is approximated by a two-exponential decay function:

$$
C_{p}(t)=A_{0}\left(a_{1} e^{-\alpha_{1} t}+a_{2} e^{-\alpha_{2} t}\right),
$$

where $A_{0}$ is the intravenously administered dose of contrast agent. The arterial input function constants were previously estimated from Gadomer plasma decay in nude mice to be $a_{1}=6.427 \mathrm{~kg} / 1, a_{2}=2.392 \mathrm{~kg} / 1, \alpha_{1}=0.2270 \mathrm{~min}^{-1}$, and $\alpha_{2}$ $=0.0258 \mathrm{~min}^{-1}$. 35

The solution to Eq. (7) is $^{24}$

$$
C_{t}(t)=K^{\text {trans }} \int_{0}^{t} C_{p}(\tau) \exp \left[-\frac{k_{\text {trans }}}{v_{e}}(t-\tau)\right] \mathrm{d} \tau,
$$

with initial conditions $C_{t}=0$ at $t=0$.

Gadomer is assumed not to enter the cells; therefore, the extravascular compartment equals EES. The volume transfer constant $K^{\text {trans }}$ was estimated by a least-squares fitting of the experimental Gadomer concentration-time curve to Eq. (9). The size of Gadomer suggests slow extravasation and no depletion of the intravascular concentration. Under such permeability-limited conditions, $K^{\text {trans }}$ equals the permeability surface area product per unit volume of tissue: ${ }^{24}$

$$
K^{\text {trans }}=\frac{P S}{V_{t}} .
$$

Permeability was also estimated by the area under the concentration curve (AUC) and the initial slope of the concentration curve. The AUC was calculated for a time period of $10 \mathrm{~min}$ post-injection to include the concentration maximum at approximately $7.5 \mathrm{~min}$ and was normalized to the background signal intensity. The initial slope was estimated from the linear fit to the five initial post-injection measurement points.

\subsection{Statistics}

A two-sample, two-tailed student's $t$-test (Minitab, Minitab, Inc., State College, Pennsylvania) assuming nonequal variances was used to perform a statistical comparison of data from normal and tumor tissue with a significance criterion of $P \leqslant 0.05$. Parameters calculated from measurements obtained in the same tumor were plotted, and linear regression was applied. For a good representation of the whole tumor, the mean CLSM values were chosen for comparison to the values obtained from DCE-MRI, which are based on the mean signal intensity in one large ROI per tumor. Tumor heterogeneity was indicated by the standard deviations of the one to three CLSM measurements in each tumor. The goodness of the linear fit was estimated by the coefficient of determination $R^{2}$, and correlations were established with a significance criterion of $P \leqslant 0.05$.

\section{Results}

\subsection{Vascular Permeability}

Extravasation of $40-\mathrm{kDa}$ dextran and Gadomer in OHS tumors and normal tissue was monitored by acquiring time series CLSM and DCE-MRI images, respectively. The dextran molecules were quickly distributed in the vasculature [Figs. 1(a) and 2(a)] and extravasated from blood vessels to the extravascular space, as detected by an enhancement of fluorescence intensity in the extravascular area [Figs. 1(b), 1(c), and 2(a)]. During the experimental time course, the signal intensity of TMR-dextran in the extravascular space increased with time, generally reached its maximum, and then slowly decreased again [Fig. 2(a)]. However, in some animals, extravasation continued throughout the total acquisition time. The intravascular concentration showed a rapid increase in intensity due to tracer distribution in the vasculature, followed by a gradual decrease due to tracer clearance and extravasation out 


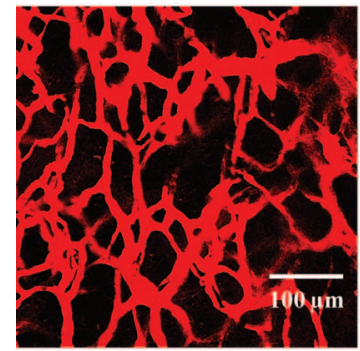

(a)

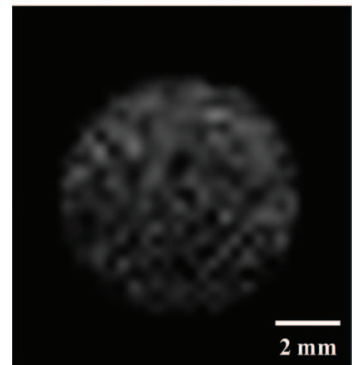

(d)

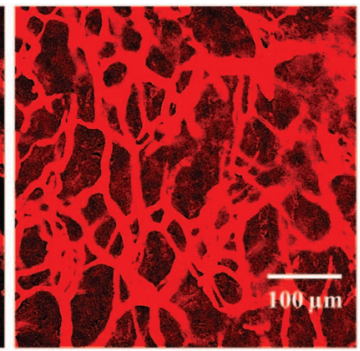

(b)

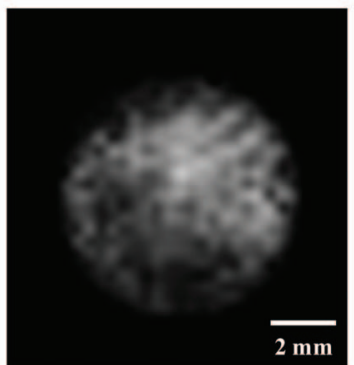

(e)

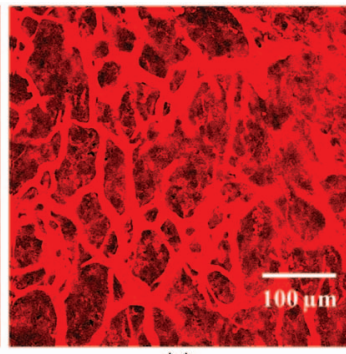

(c)

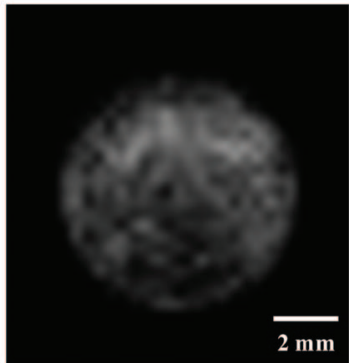

(f)

Fig. 1 Dynamic time series images of OHS tumors in a dorsal window chamber in mice, acquired by CLSM [(a) to (c)] and DCE-MRI [(d) to (f)]. CLSM images acquired at $t=2 \mathrm{~min}(\mathrm{a}), t=9 \mathrm{~min}(\mathrm{~b})$, and $t=25 \mathrm{~min}$ (c) after injection show fluorescence intensity enhancement due to extravasation of 40-kDa TMR-dextran from blood vessels and accumulation in the extravascular space. Images from DCE-MRI time series at pre-contrast (d) and at $t=5 \mathrm{~min}(\mathrm{e})$ and $t=37 \mathrm{~min}(\mathrm{f})$ after Gadomer injection show accumulation of the contrast agent in the tumor tissue. The pixel sizes were $0.9 \times 0.9 \mu \mathrm{m}^{2}$ and $578 \times 578 \mu \mathrm{m}^{2}$ for CLSM and DCE-MRI, respectively.

of blood vessels [Fig. 2(a)]. The kinetics of the extra- and intravascular concentrations varied in different tumor regions and between different tumors. The ratio of intra- to extravascular intensity were plotted against a modified time axis in a Patlak plot [Fig. 2(b)], and the terminal slope of the fit indicated the extravasation rate $K_{i}{ }^{32}$ For a 35-min time course, a straight line was generally well fitted to the terminal slope, and the extravasation rate $K_{i}$ was significantly different for tumor and normal tissues. However, the terminal slope for normal tissue was negative (data not shown); thus, normal tissue was excluded from further analysis. Mean values and standard deviations of $K_{i}$ and apparent permeability $P$ for tumor tissue are listed in Table 1.

Pre-contrast [Fig. 1(d)] and post-contrast [Figs. 1(e) and 1(f)] DCE-MRI images were acquired for the entire chamber window. At 5 min post-contrast [Fig. 1(e)], signal enhancement due to Gadomer accumulation in the tissue was observed. For all animals, the signal intensity had nearly returned to the pre-contrast level after $37 \mathrm{~min}$ [Fig. 1(f)]. Kinetic parameters were estimated from the DCE-MRI data by fitting the Tofts model to the Gadomer concentration-time curves. A typical tissue concentration-time curve is shown in Fig. 2(c). Gadomer extravasated from blood vessels into the EES and the concentration-time curves reflect combined clearance and accumulation of the contrast agent in the tumor tissue. The Gadomer concentration increased and reached a maximum after an average of $7.5 \mathrm{~min}$ and then subsequently decreased due to clearance from both plasma and the extravascular space. The area under the concentration curve AUC and the initial slope of the concentration curve were

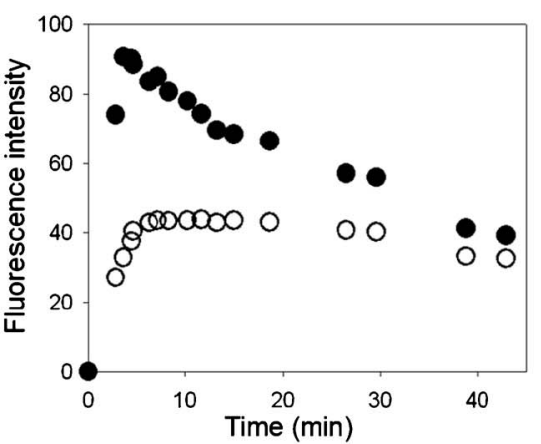

(a)

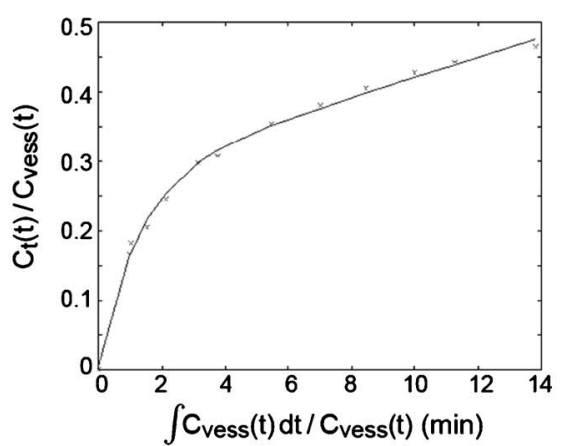

(b)

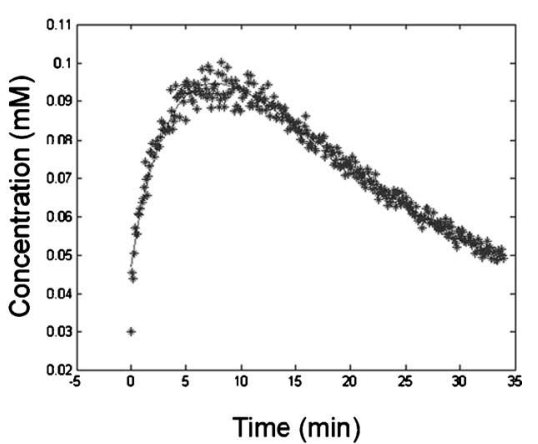

(c)

Fig. 2 Dynamic measurements of tumor permeability. (a) CLSM time series showing intravascular (solid circles) and extravascular (open circles) fluorescence intensity of 40-kDa TMR-dextran as a function of time after injection. (b) Patlak plot from CLSM time series images. (c) Tofts model fitted to DCE-MRI curve showing concentration versus time after Gadomer injection. 
Reitan et al.: Characterization of tumor microvascular structure and permeability...

Table 1 Estimates of permeability in tumor tissue in dorsal window chambers. $^{\text {a }}$

\begin{tabular}{|c|c|}
\hline Vascular permeability & Tumor \\
\hline \multicolumn{2}{|l|}{ CLSM: } \\
\hline Extravasation rate $K_{i}\left(10^{-4} \mathrm{~s}^{-1}\right)$ & $0.39 \pm 0.19$ \\
\hline $\begin{array}{l}\text { Apparent permeability } P \\
\left(10^{-7} \mathrm{~cm}^{-1} \mathrm{~s}^{-1}\right)\end{array}$ & $2.80 \pm 1.41$ \\
\hline \multicolumn{2}{|l|}{ DCE-MRI: } \\
\hline Time-to-peak TTP (s) & $7.47 \pm 1.41$ \\
\hline EES volume fraction $v_{e}$ & $0.20 \pm 0.03$ \\
\hline Area under the curve $A U C$ (min) & $9.72 \pm 2.08$ \\
\hline $\begin{array}{l}\text { Volume transfer constant } K^{\text {trans }} \\
\left(10^{-2} \mathrm{~min}^{-1}\right)\end{array}$ & $2.80 \pm 0.90$ \\
\hline Initial slope $\left(10^{-3} \mathrm{mM} / \mathrm{min}\right)$ & $2.40 \pm 0.92$ \\
\hline $\begin{array}{l}\text { CLSM parameters are represented by } \\
\text { ased on } n=5 \text { mice with tumor and one } \\
\text { hean DCE-MRI values are based on } n=6 \\
\text { er tumor. }\end{array}$ & $\begin{array}{l}\text { standard dev } \\
\text { and onts per tum } \\
\text { and one }\end{array}$ \\
\hline
\end{tabular}

extracted directly from experimental data. The mean native $T_{1}$ values for all tumors were measured to $T_{1}=1647 \mathrm{~ms}$. No significant differences was found between normal and tumor tissue for the volume transfer constant $K^{\text {trans }}$ and the volume fraction $v_{e}$, and only mean values and standard deviations for tumor tissue are therefore listed in Table 1.

\subsection{Microvascular Structure}

Structural parameters, including fractal dimension, number of branching points, and vascular density, were quantified (Table 2) from CLSM images. All parameter values were significantly higher for tumor vasculature than for normal vasculature. This difference was also demonstrated by the striking difference between the tortuous tumor vessels [Fig. 3(a)] and the well-organized normal blood vessels [Fig. 3(b)]. To obtain a high spatial resolution for the MR images of blood vessels, a long acquisition time was required, and the larger intravas-
Table 2 Estimates of microvascular structure in tumors and normal tissue in dorsal window chambers. ${ }^{\text {a }}$

\begin{tabular}{lcc}
\hline Microvascular structure & Tumor & Control \\
\hline Fractal dimension & $1.73 \pm 0.05$ & $1.58 \pm 0.03$ \\
Vascular density & $0.21 \pm 0.05$ & $0.10 \pm 0.02$ \\
Number of branching points & $160.4 \pm 55.6$ & $50.7 \pm 19.0$ \\
Vascular surface area & & \\
& & \\
per tissue volume $S / V_{t}\left(\mathrm{~cm}^{-1}\right)$ & $151.4 \pm 40.9$ & $95.7 \pm 25.9$ \\
\hline $\begin{array}{l}\text { a CLSM parameters are represented by mean values and standard deviations } \\
\text { based on } n=6 \text { mice with tumor and } 6 \text { controls, and one to three measurements } \\
\text { per mouse. }\end{array}$
\end{tabular}

cular contrast agent albumin-(Gd-DTPA) was used. Larger blood vessels were clearly visualized [Fig. 3(c)].

\subsection{Correlation of Vascular Structural and Functional Parameters Measured by CLSM and DCE-MRI}

The permeability indicators and structural parameters estimated by CLSM and DCE-MRI were plotted and correlated, and a linear regression was applied to the data (Fig. 4). A high degree of positive correlation $(P<0.001)$ was found between the various structural vascular parameters, demonstrating that a higher fractal dimension implies a higher number of branching points and vascular density. The structural parameters were significantly higher in tumors than in normal tissue, which can be observed from the two distinct populations in Figs. 4(a) and 4(b). Increased extravasation, as measured by CLSM and represented by the extravasation rate $K_{i}$, correlated $(P \leqslant 0.02)$ with a higher fractal dimension and vascular density [Figs. 4(c) and 4(d)]. $K^{\text {trans }}$, estimated from DCE-MRI, was shown to correlate $(P=0.04)$ with $K_{i}$ [Fig. 4(e) ]. However, no correlation was found between $K^{\text {trans }}$ and apparent permeability $P$, which relates $K_{i}$ to the vascular surface area [Fig. 4(f)]. $K_{i}$ correlated significantly $(P=0.008)$ with the initial slope of the DCE-MRI concentration curves [Fig. 4(h)], but the correlation between $K_{i}$ and AUC [Fig. 4(g)] was not significant $(P=0.09)$.

The CLSM data in Fig. 4 show the mean values from one to three measurements at different locations in the tissue of

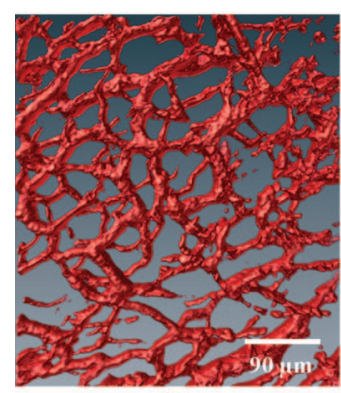

(a)

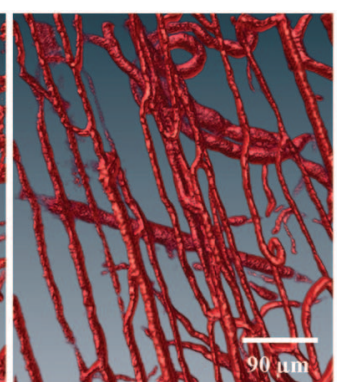

(b)

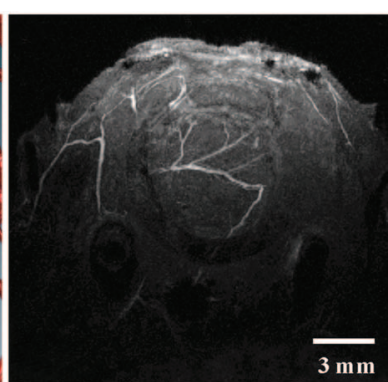

(c)

Fig. 3 Blood vessels in a dorsal window chamber were imaged by CLSM [(a) and (b)] and MRI (c). (a) Abnormal, tortuous tumor vessels and (b) well-organized normal vessels visualized in Amira by 3-D volume rendering of CLSM Z-stacks of tissue after 2-MDa FITC-dextran injection. (c) Maximum-intensity projection of MRI Flash 3-D sequence for imaging of blood vessels. Albumin-(Gd-DTPA) was used as an MRI contrast agent. 
Reitan et al.: Characterization of tumor microvascular structure and permeability...

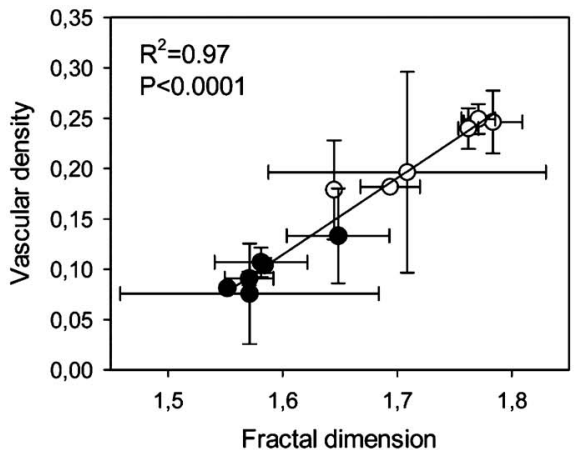

(a)

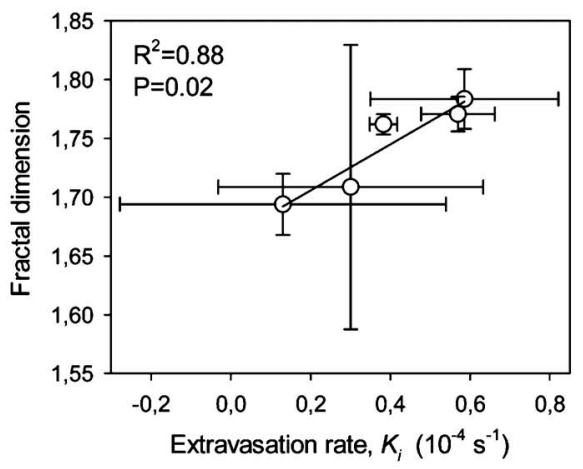

(c)

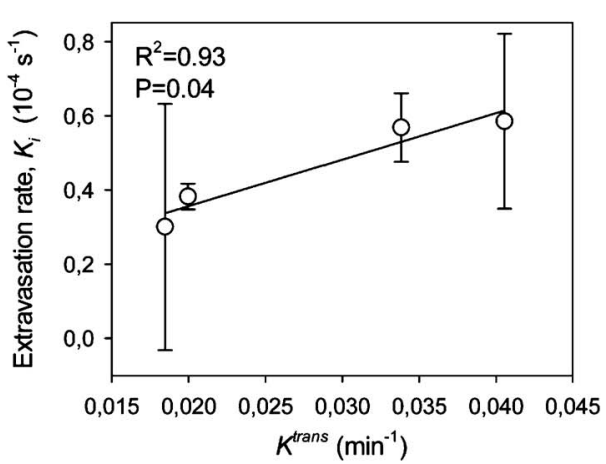

(e)

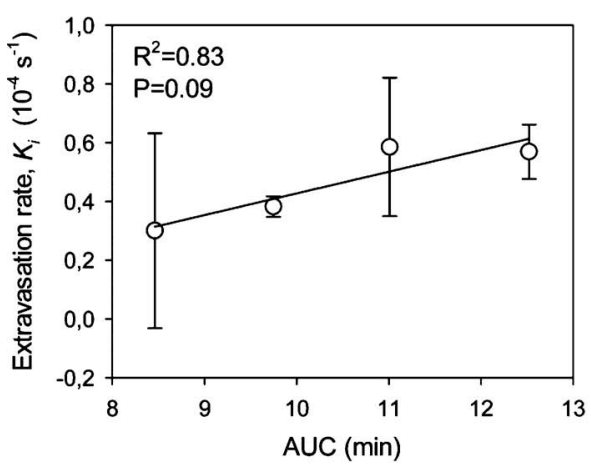

(g)

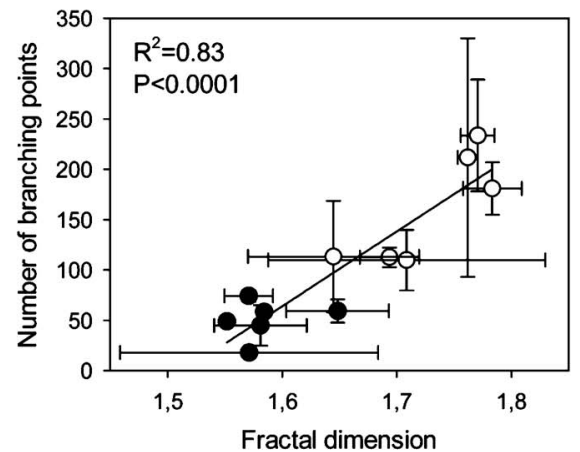

(b)

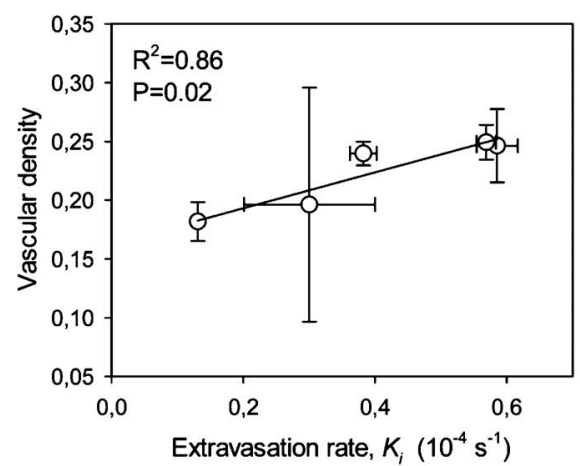

(d)

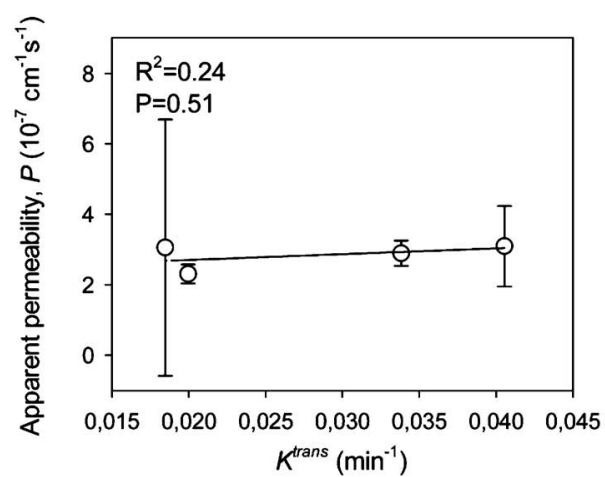

(f)

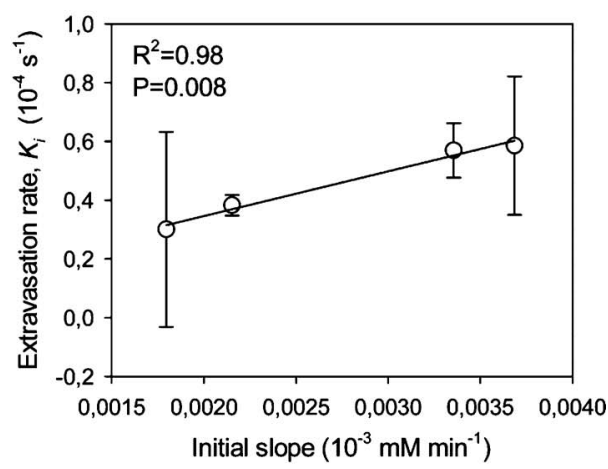

(h)

Fig. 4 Correlation analysis of microvascular structural parameters and permeability indicators obtained with CLSM and DCE-MRI. The microvascular structure (fractal dimension, vascular density, and number of branching points) was measured by CLSM for normal (solid circles) and tumor tissue (open circles). Permeability parameters, as measured by CLSM (extravasation rate $K_{i}$ and apparent permeability $P$ ) and DCE-MRI ( $K^{\text {trans }}$, AUC, and initial slope), were plotted for tumor tissue. (a) and (b) Relationship between microvascular structural parameters. (c) and (d) $K_{i}$ versus microvascular structure. (e) to (h) Relationship between permeability parameters obtained by CLSM and DCE-MRI. Permeability was successfully measured with both CLSM and DCE-MRI for four mice. Mean values and standard deviations are based on measurements in one to three ROIs in one mouse. The goodness of linear fit $R^{2}$ and $P$-values are given in each plot. The number of measurement points in each graph corresponds to the number of animals for which the respective parameters were successfully acquired. 
each mouse. Each data point represents one mouse. To optimize the signal-to-noise ratio (SNR) for the DCE-MRI data, only one ROI per animal was analyzed. The heterogeneity within each tumor, which was observed with CLSM, is indicated by the standard deviations in Fig. 4. In particular, $K_{i}$ showed large variations at the three measured locations, with the highest coefficient of variation (standard deviation/mean) at 2.43 for a tumor.

\section{Discussion}

Tumor microvasculature is characterized by abnormal structures and enhanced permeability. These properties may predict therapeutic response. ${ }^{6,36-41}$ Robust methods are therefore required to quantify the structural and functional parameters of tumor vasculature. In the present study, a correlation between microvascular structure and permeability indicators was found in tumor tissue growing in dorsal window chambers. Few previous studies have directly related the permeability of tumor vessels to tumor microvascular structure by applying these techniques in combination. ${ }^{42}$

MRI provides noninvasive images of vasculature and is clinically important for diagnosis and the monitoring of therapeutic outcomes. However, MRI has limited spatial resolution. In contrast, confocal imaging, with a typical spatial resolution of 200 to $300 \mathrm{~nm}$, provides detailed information about microvasculature that is not accessible with MRI. In the present study, larger vessels were clearly observed using MRI, while small capillaries and their branching patterns were visualized using CLSM. CLSM thus gives more detailed information about local variations than MRI. In addition, CLSM can demonstrate whether MRI contrast agents are confined to the vasculature or also located in the EES.

\subsection{Choice of Molecular Tracers}

Gadomer was chosen as an MRI contrast agent because it is biocompatible, has previously been shown to provide information about vascular permeability, ${ }^{23,35,43,44}$ and has a suitable size for the present experiments. Gadomer could not be fluorescently labeled, as all of its amines were occupied by Gd. A similarly sized, well characterized dextran was therefore chosen as a fluorescent counterpart to Gadomer. Multimodal macromolecular contrast agents, such as albumin-(Gd-DTPA) were considered, but they are generally larger than the size required for this study.

Dextrans have previously been used to study permeability in tumors growing in dorsal window chambers. Dreher et al. ${ }^{45}$ used dextrans of various molecular weights (3.3 $\mathrm{kDa}$ to $2 \mathrm{MDa}$ ) and showed that maximal accumulation occurred using dextrans with molecular weights of 40 to $70 \mathrm{kDa}$, indicating that the enhanced permeability effect is relevant for the chosen molecular tracers in the present study. Reyes-Aldasoro et al. ${ }^{32}$ used 40-kDa dextran to characterize the permeability of capillaries in P22 sarcomas growing in dorsal window chambers of rats.

\subsection{Permeability Estimated by CLSM and DCE-MRI}

All extravascular fluorescence intensity curves of $40-\mathrm{kDa}$ dextran, as measured by CLSM, showed an initial increase before the intensity stabilized and even occasionally decreased, most likely due to reabsorbance into the vascular space or oozing out of the tumor due to the outward convective gradient. There were large variations in the kinetics and maximum values of the fluorescence intensity, both within a given tumor and between tumors in different animals, demonstrating heterogeneous permeability. The spatial heterogeneity of extravasation in tumors is well known and is due to heterogeneous blood supply and anatomic variations of tumor vessels. $^{16,46,47}$ The correlation between functional and anatomic heterogeneity has been demonstrated by Dvorak et al., ${ }^{46}$ who showed that different types of vessels exhibit different degrees of leakiness.

Gadomer permeability was estimated from DCE-MRI measurements. The kinetics of the concentration curves obtained in the present study were consistent with previously reported contrast uptake curves of Gadomer. ${ }^{35}$ The steep initial slope indicates high vascular permeability. The fast initial decay of the curves indicates that Gadomer is being efficiently cleared from plasma by glomerular filtration, resulting in a low concentration of Gadomer in the tissue, which is consistent with a low maximum value of the concentration curve.

For kinetic modeling of the CLSM extravasation data, the method developed by Patlak et al. ${ }^{31}$ was chosen. DCE-MR images were analyzed by the well-established, twocompartment kinetic model developed by Tofts et al. ${ }^{24}$ Previously measured arterial input constants ${ }^{35}$ and $\mathrm{T}_{1}$ relaxivity gave acceptable fits to the DCE-MRI data. The Patlak method used to analyze the CLSM data is based on measured fluorescence intensity values and requires direct measurement of vascular intensity. This method avoids the use of complex pharmacokinetic modeling and does not require input parameters such as the arterial input function used in the Toft model. Significant differences between extravasation in tumors and normal tissue were observed using the Patlak model. The terminal slope of the Patlak plots assumed zero or negative values for normal tissue, indicating rapid clearance via the lymphatic system, whereas the tracers remained in the interstitium of tumors for a longer time period due to a depressed lymphatic system. Because of the negative slope of the Patlak plot, normal tissue failed to fulfill the requirement of irreversible leakage from the plasma compartment to the extravascular space; thus, measurements of normal tissue were not included in further analyses.

The fact that CLSM but not DCE-MRI could discriminate between tumor and normal tissue may be explained by differences in experimental setup and/or data analysis. Vascular permeability has previously been shown to depend on molecular weight and charge of molecular tracers. ${ }^{45,48}$ However, although there is a charge difference (dextran is neutral and Gadomer has a charge of -2 in solution), ${ }^{49} 40-\mathrm{kDa}$ dextran and Gadomer are approximately equal in size. Another possible explanation may be the different-sized ROIs used for the two techniques. The tissue in the dorsal window chamber was thin (approximately $0.5 \mathrm{~mm}$ for tumors and $<0.5 \mathrm{~mm}$ for normal tissue), and the SNR in the images acquired with DCE-MRI was low. To maximize the SNR, the analyzed ROI constituted the entire volume in the chamber window with detectable signal enhancement. Thus, the tissue in the ROI may have contained a mix between intra- and extravascular space, as well as included normal blood vessels and heterogeneous tumor vasculature. Because the signal intensity in the DCE-MRI data is the mean intensity throughout the ROI vol- 
ume, the possible mixture of tissue types may have caused undistinguishable tumor permeability between tumor mice and controls. In CLSM, on the other hand, only ROIs with tumor vasculature was analyzed. The use of several smaller ROIs to analyze DCE-MRI data-e.g., pixel-based DCEMRI-would be desirable, but this requires a higher SNR than was achievable with the present experimental setup. Strategies for future experiments may be to increase the SNR and the spatial resolution at the cost of a lower time resolution.

\subsection{Correlation between CLSM and DCE-MRI}

The positive correlation obtained between $K^{\text {trans }}$ of Gadomer, as measured by DCE-MRI, and the extravasation rate $K_{i}$ of 40-kDa dextran, as measured by CLSM, demonstrated that extravasation values measured by these two methods reflected each other despite the fact that different molecules and different mathematical models were used. The total vascular surface area $S$ was estimated, and the apparent permeability per vascular surface area $P$ was calculated from $K_{i}$ by Eq. (4). However, $P$ did not correlate with structural parameters (data not shown) or with $K^{\text {trans }}$ [Fig. 4(f)]. This may be because $S$ was not well defined in the optical section, as the optical section was thinner than the diameters of most vessels. In another study of extravasation of $40-\mathrm{kDa}$ dextran in tumors using the dorsal window chamber model, Reyes-Aldasoro et al. $^{32}$ estimated extravasation rate and apparent permeability from 3-D multiphoton images with the Patlak model. Such 3-D images of one ROI may provide a better defined vascular surface area. However, in the present work, we aimed to demonstrate the heterogeneity within the vasculature by measuring three different ROIs. The time series required a high time resolution due to relatively fast extravasation. Therefore, we chose to apply 2-D imaging of several ROIs instead of 3-D acquisition of one ROI.

Quantitative data for permeability was not the main focus of the present work and would have required thorough calibration of concentration versus intensity. Instead, we focused on relating information obtained from different techniques and analyses. A significant correlation was found between $K_{i}$ and the initial slope of the DCE-MRI concentration curve, both of which are permeability indicators. With a slightly less stringent significance level $(P=0.09), K_{i}$ also correlated with AUC. This indicates that a variety of relevant parameters may be applied in complementary CLSM and MRI analyses.

\subsection{Relationship between Microvascular Structure and Permeability}

The CLSM images revealed several structural parameters that correlated with each other and with the estimated extravasation value of dextran. In addition, significant differences were found between the microvascular structure of tumors and normal tissue. The CLSM ROIs for tumors were carefully chosen for analysis of angiogenic vasculature. Because of the rapidly expanding, chaotic, and branched nature of angiogenic vessels, the complexity of the vasculature (as indicated by increased fractal dimension, number of branching points, and vascular density) was higher for tumors than for normal vessels. Vascular endothelial growth factor (VEGF), which is responsible for angiogenesis, is also a strongly permeabilizing agent. Therefore, the leaky blood vessels are neoformed, angiogenic microvessels, and the positive correlation between permeability and tumor vascular structure was expected. ${ }^{50}$

Fractal analysis provides an overall mathematical description of the vascular network and may reflect the degree of vascular tortuosity or self-similarity. Tumor vasculature contains a variety of vessel types; arteries, veins, and capillaries cannot be clearly distinguished. ${ }^{10}$ The formation of new blood vessels in a tumor by angiogenesis is essential for tumor growth and metastasis, and the fractal dimension has been shown to yield information about vascular architecture, tumor growth, and angiogenesis. ${ }^{18}$ A previous study by Gazit et al. ${ }^{20}$ showed that the fractal dimension was dependent on the vessel type and assumed different values for tumor vessels, the normal arteriovenous network, and the capillary network. The fractal dimension in the present study assumed higher values for chaotic tumor vessels than for normal tissue. The normal vasculature was well organized, although different types of vessels (mainly thin parallel capillaries, but also larger vessels) were observed. The mean fractal dimension for tumor tissues correlated positively with the extravasation rate $K_{i}$, the number of branching points, and vascular density, all of which may be potential indicators of angiogenic activity.

Vascular parameters such as microvascular density and permeability may serve as prognostic factors and have been shown to predict outcome in patients with breast, colon, and lung cancers. ${ }^{6,36-39}$ Anti-angiogenic therapy that blocks VEGF or its receptor (VEGFR2) has been reported to induce apoptosis in endothelial cells and decrease vessel diameter, density, and permeability. ${ }^{40,41}$ These changes were accompanied by the development of a normal vessel structure, which resulted in decreased interstitial fluid pressure and a more normal microenvironment. ${ }^{40,41}$ The normalization of the vasculature and the reduced interstitial fluid pressure may facilitate drug delivery. There is a need for reliable biomarkers that reflect the vascular structure and, therefore, the prognostic outcome. Vascular permeability may be such a marker, and $K^{\text {trans }}$ is reported to be uniquely suited for evaluating the normalization process of tumor vasculature. ${ }^{51}$ The present work demonstrates that MRI and CLSM may be useful complementary methods for the characterization of permeability.

\section{Conclusion}

By combining CLSM and DCE-MRI, we measured permeability and microvascular architecture in tumors growing in dorsal window chambers in mice. A correlation was found between permeability parameter values that were estimated by the two techniques. Permeability was further shown to depend on structural parameters such as fractal dimension and vascular density. We have demonstrated that the dorsal window tumor model gives a unique opportunity to use CLSM and MRI as complementary techniques. CLSM can both validate results obtained with MRI and provide insights into the spatial heterogeneous microenvironment at a microscopic level, which is not possible with MRI alone.

\section{Acknowledgments}

We gratefully acknowledge Kristin Sæterbø, Dept. of Physics, NTNU, for growing cells and implanting chambers and tumors; Tina Bugge Pedersen and Else Marie Huuse, Dept. of 
Circulation and Medical Imaging, NTNU, for assistance in the lab; and Wenche Strand, Dept. of Biotechnology, NTNU, for synthesizing albumin-(Gd-DTPA). We also thank Christian Brekken, Dept. of Circulation and Medical Imaging, NTNU (previous address), for valuable discussions. The contrast agent Gadomer was kindly provided by Bayer Schering Pharma AG, Berlin. This work was supported by the Norwegian Cancer Society.

\section{References}

1. P. Vaupel and A. Mayer, "Hypoxia and anemia: effects on tumor biology and treatment resistance," Transfus. Clin. Biol. 12, 5-10 (2005).

2. D. Fukumura and R. K. Jain, "Tumor microenvironment abnormalities: causes, consequences, and strategies to normalize," J. Cell. Biochem. 101, 937-949 (2007)

3. G. U. Dachs and D. J. Chaplin, "Microenvironmental control of gene expression: implications for tumor angiogenesis, progression, and metastasis," Semin. Radiat. Oncol. 8, 208-216 (1998).

4. R. K. Jain, "Delivery of molecular medicine to solid tumors: lessons from in vivo imaging of gene expression and function," Cytokines Cell Mol. Ther. 74, 7-25 (2001).

5. S. H. Jang, M. G. Wientjes, D. Lu, and J. L.-S. Au, "Drug delivery and transport to solid tumors," Pharm. Res. 20, 1337-1350 (2003).

6. S. Kumar, A. Ghellal, C. Li, G. Byrne, N. Haboubi, J. M. Wang, and N. Bundred, "Breast carcinoma: vascular density determined using CD105 antibody correlates with tumor prognosis," Cancer Res. 59, 856-861 (1999).

7. A. I. Minchinton and I. F. Tannock, "Drug penetration in solid tumours," Nat. Rev. Cancer 6, 583-592 (2006).

8. P. Sonveaux, C. Dessy, A. Brouet, B. H. Jordan, V. Grégoire, B. Gallez, J.-L. Balligand, and O. Feron, "Modulation of the tumor vasculature functionality by ionizing radiation accounts for tumor radiosensitization and promotes gene delivery," FASEB J. 16, 1979(2002).

9. P. Vaupel, F. Kallinowski, and P. Okunieff, "Blood flow, oxygen and nutrient supply, and metabolic microenvironment of human tumors: a review," Cancer Res. 49, 6449-6465 (1989).

10. R. K. Jain, "Determinants of tumor blood-flow-a review," Cancer Res. 48, 2641-2658 (1988).

11. J. R. Less, T. C. Skalak, E. M. Sevick, and R. K. Jain, "Microvascular architecture in a mammary-carcinoma-branching patterns and vessel dimensions," Cancer Res. 51, 265-273 (1991).

12. M. Leunig, F. Yuan, M. D. Menger, Y. Boucher, A. E. Goetz, K. Messmer, and R. K. Jain, "Angiogenesis, microvascular architecture, microhemodynamics, and interstitial fluid pressure during early growth of human adenocarcinoma 1s174t in scid mice," Cancer Res. 52, 6553-6560 (1992).

13. F. Yuan, "Transvascular drug delivery in solid tumors," Semin. Radiat. Oncol. 8, 164-175 (1998).

14. L. Eikenes, Ø. S. Bruland, C. Brekken, and C. de L. Davies, "Collagenase increases the transcapillary pressure gradient and improves the uptake and distribution of monoclonal antibodies in human osteosarcoma xenografts," Cancer Res. 64, 4768-4773 (2004).

15. Y. Boucher, L. T. Baxter, and R. K. Jain, "Interstitial pressure gradients in tissue-isolated and subcutaneous tumors-implications for therapy," Cancer Res. 50, 4478-4484 (1990).

16. H. Lyng, A. O. Vorren, K. Sundfør, I. Taksdal, H. H. Lien, O. Kaalhus, and E. K. Rofstad, "Intra- and intertumor heterogeneity in flood perfusion of human cervical cancer before treatment and after radiotherapy," Int. J. Cancer 96, 182-190 (2001).

17. D. Mancardi, G. Varetto, E. Bucci, F. Maniero, and C. Guiot, "Fractal parameters and vascular networks: facts and artifacts," Theor. Biol. Med. Mod. 5(1), 12 (2008).

18. J. W. Baish and R. K. Jain, "Fractals and cancer," Cancer Res. 60 , 3683-3688 (2000).

19. E. Sabo, A. Boltenko, Y. Sova, A. Stein, S. Kleinhaus, and M. B. Resnick, "Microscopic analysis and significance of vascular architectural complexity in renal cell carcinoma," Clin. Cancer Res. 7, 533$537(2001)$

20. Y. Gazit, D. A. Berk, M. Leunig, L. T. Baxter, and R. K. Jain, "Scaleinvariant behavior and vascular network formation in normal and tumor tissue," Phys. Rev. Lett. 75, 2428-2431 (1995).
21. H. C. Roberts, M. Saeed, T. P. L. Roberts, A. Mühler, D. M. Shames, J. S. Mann, M. Stiskal, F. Demsar, and R. C. Brasch, "Microscopic analysis and significance of vascular architectural complexity in renal cell carcinoma," J. Magn. Reson Imaging 7, 331-338 (1997).

22. J. R. Ewing, S. L. Brown, M. Lu, S. Panda, G. Ding, R. A. Knight, Y. Cao, Q. Jiang, T. N. Nagaraja, J. L. Churchman, and J. D. Fenstermacher, "Model selection in magnetic resonance imaging measurements of vascular permeability: Gadomer in a 9L model of rat cerebral tumor," J. Cereb. Blood Flow Metab. 26, 310-320 (2006).

23. M. Verhoye, B. P. J. van der Sanden, P. F. J. W. Rijken, H. P. W. Peters, A. J. Van der Kogel, G. Pee, G. Vanhoutte, A. Heerschap, and A. Van der Linden, "Assessment of the neovascular permeability in glioma xenografts by dynamic T-1 MRI with Gadomer-17," Magn Reson. Med. 47, 305-313 (2002).

24. P. S. Tofts, G. Brix, D. L. Buckley, J. L. Evelhoch, E. Henderson, M. V. Knopp, H. B. W. Larsson, T.-Y. Lee, N. A. Mayr, G. J. M. Parker, R. E. Port, J. Taylor, and R. M. Weisskoff, "Pharmacokinetics of Gadomer-17, a new dendritic magnetic resonance contrast agent," $J$. Magn. Reson Imaging 10, 223-232 (1999).

25. R. Weissleder and M. J. Pittet, "Imaging in the era of molecular oncology," Nature 452, 580-589 (2008).

26. A. Erikson, I. Tufto, A. B. Bjønnum, Ø. S. Bruland, and C. de L. Davies, "The impact of enzymatic degradation on the uptake of differently sized therapeutic molecules," Anticancer Res. 28, 3557-3566 (2008).

27. Ø. Fodstad, A. Brøgger, Ø. Bruland, Ø. P. Solheim, J. M. Nesland, and A. Pihl, "Characteristics of a cell-line established from a patient with multiple osteosarcoma, appearing 13 years after treatment for bilateral retinoblastoma," Int. J. Cancer 38, 33-40 (1986).

28. B. Misselwitz, H. Schmitt-Willich, W. Ebert, T. Frenzel, and H. J. Weinmann, "Pharmacokinetics of Gadomer-17, a new dendritic magnetic resonance contrast agent," Magn. Reson. Mater. Phys., Biol. Med. 12, 128-134 (2001).

29. H. J. Weinmann, W. Ebert, B. Misselwitz, and H. Schmitt-Willich, "Tissue-specific MR contrast agents," Eur. J. Radiol. 46, 33-44 (2003).

30. M. D. Ogan, "Albumin labeled with Gd-DTPA: an intravascular contrast-enhancing agent for magnetic resonance blood pool imaging: preparation and characterization," Invest. Radiol. 23, 961-961 (1988).

31. C. S. Patlak, R. G. Blasberg, and J. D. Fenstermacher, "Graphical evaluation of blood-to-brain transfer constants from multiple-time uptake data," J. Cereb. Blood Flow Metab. 3, 1-7 (1983).

32. C. C. Reyes-Aldasoro, I. Wilson, V. E. Prise, P. R. Barber, S. M. Ameer-Beg, B. Vojnovic, V. J. Cunningham, and G. M. Tozer, "Estimation of apparent tumor vascular permeability from multiphoton fluorescence microscopic images of P22 rat sarcomas in vivo," Microcirculation 15, 65-79 (2008).

33. U. Bickel, "How to measure drug transport across the blood-brain barrier," Neurotherapeutics 2, 15-26 (2005).

34. Bayer Schering Pharma AG, Berlin, Private Communication (2009).

35. M. Verhoye, P. J. van der S. Boudewijn, P. Rijken, H. Peters, A. J. Van der Kogel, G. Pée, G. Vanhoutte, A. Heerschap, and A. Van der Linden, "Quantification of tumor microvascular permeability in human glioma xenografts using dynamic T1 MRI with Gadomer-17," Proc. SPIE 3660, 418-428 (1999).

36. N. Weidner, J. Folkman, F. Pozza, P. Bevilacqua, E. N. Allred, D. H. Moore, S. Meli, and G. Gasparini, "Tumor angiogenesis-a new significant and independent prognostic indicator in early-stage breast carcinoma," J. Natl. Cancer Inst. 84, 1875-1887 (1992).

37. P. B. Vermeulen, D. Verhoeven, G. Hubens, E. Vanmarck, G. Goovaerts, M. Huyghe, E. A. Debruijn, A. T. Vanoosterom, and L. Y. Dirix, "Microvessel density, endothelial-cell proliferation and tumorcell proliferation in human colorectal adenocarcinomas," Ann. Oncol. 6, 59-64 (1995).

38. L. M. Ellis, Y. Takahashi, C. J. Fenoglio, K. R. Cleary, C. D. Bucana, and D. B. Evans, "Vessel counts and vascular endothelial growth factor expression in pancreatic adenocarcinoma," Eur. J. Cancer 34 337-340 (1998)

39. I. G. Duarte, B. L. Bufkin, M. F. Pennington, A. A. Gal, C. Cohen, A. S. Kosinski, K. A. Mansour, and J. I. Miller, "Angiogenesis as a predictor of survival after surgical resection for stage I non-small-cell lung cancer," J. Thorac. Cardiovasc. Surg. 115, 652-658 (1998).

40. Y. Tsuzuki, D. Fukumura, B. Oosthuyse, C. Koike, P. Carmeliet, and R. K. Jain, "Vascular endothelial growth factor (VEGF) modulation 
by targeting hypoxia-inducible factor- 1 alpha $\rightarrow$ hypoxia response element $\rightarrow$ VEGF cascade differentially regulates vascular response and growth rate in tumors," Cancer Res. 60, 6248-6252 (2000).

41. F. Yuan, Y. Chen, M. Dellian, N. Safabakhsh, N. Ferrara, and R. K. Jain, "Quantification of tumor microvascular permeability in human glioma xenografts using dynamic T1 MRI with Gadomer-17," Proc. Natl. Acad. Sci. U.S.A. 93, 14765-14770 (1996).

42. J. V. Gaustad, K. G. Brurberg, T. G. Simonsen, C. S. Mollatt, and E. K. Rofstad, "Tumor vascularity assessed by magnetic resonance imaging and intravital microscopy imaging," Neoplasia 10, 354-362 (2008).

43. R. Duncan and L. Izzo, "Dendrimer biocompatibility and toxicity," Adv. Drug Delivery Rev. 57, 2215-2237 (2005).

44. M. Y. Su, A. Mühler, X. Lao, and O. Nalcioglu, "Tumor characterization with dynamic contrast-enhanced MRI using MR contrast agents of various molecular weights," Magn. Reson. Med. 39, 259269 (1998).

45. M. R. Dreher, W. G. Liu, C. R. Michelich, M. W. Dewhirst, F. Yuan, and A. Chilkoti, "Tumor vascular permeability, accumulation, and penetration of macromolecular drug carriers," J. Natl. Cancer Inst. 98, 335-344 (2006).

46. H. F. Dvorak, J. A. Nagy, J. T. Dvorak, and A. M. Dvorak, "Identi- fication and characterization of the blood-vessels of solid tumors that are leaky to circulating macromolecules," Am. J. Pathol. 133, 95-109 (1988).

47. R. K. Jain, "Vascular and interstitial barriers to delivery of therapeutic agents in tumors," Cancer Metastasis Rev. 9, 253-266 (1990).

48. M. Dellian, F. Yuan, V. S. Trubetskoy, V. P. Torchilin, and R. K. Jain, "Vascular permeability in a human tumour xenograft: molecular charge dependence," Br. J. Cancer 82, 1513-1518 (2000).

49. C. Nguyen-Minh, V. M. Haughton, H. S. An, J. W. You, S. Wook, and K.-C. Ho, "Contrast media of high and low molecular weights in the detection of recurrent herniated disks," AJNR Am. J. Neuroradiol. 19 889-893 (1998).

50. J. A. Nagy, E. Vasile, D. Feng, X. Sundberg, L. F. Brown, M. J. Detmar, J. A. Lawitts, L. Benjamin, X. Tan, E. J. Manseau, A. M. Dvorak, and H. F. Dvorak, "Vascular permeability factor/vascular endothelial growth factor induces lymphangiogenesis as well as angiogenesis," J. Exp. Med. 196, 1497-1506 (2002).

51. M. M. Pike, C. N. Stoops, C. P. Langford, N. S. Akella, L. B. Nabors, and G. Y. Gillespie, "High-resolution longitudinal assessment of flow and permeability in mouse glioma vasculature: sequential small molecule and SPIO dynamic contrast agent MRI," Magn. Reson. Med. 61, 615-625 (2009). 